\title{
Some Results on Weakly Symmetric Kenmotsu Manifolds
}

\author{
J. P. Singh ${ }^{1}$ and K. Lalnunsiami ${ }^{2 *}$ \\ Department of Mathematics \& Computer Science, Mizoram University, \\ Aizawl-796004, Mizoram, India \\ E-mail: *siamiofficial@gmail.com
}

\begin{abstract}
In this paper, we investigate weakly symmetric, weakly Ricci symmetric, weakly concircular symmetric and weakly concircular Ricci symmetric properties of a Kenmotsu manifold admitting a semi-symmetric metric connection. Some results on weakly -projectively symmetric Kenmotsu manifold with respect to a semi-symmetric metric connection are obtained. An example of a weakly symmetric and weakly Ricci symmetric Kenmotsu manifold with respect to this connection is constructed.
\end{abstract}

Keywords: Weakly Ricci Symmetric Manifold, Weakly Concircular Symmetric Manifold, Weakly Concircular Ricci Symmetric Manifold, $m$-Projective Curvature Tensor, Weakly $m$-Projectively Symmetric Manifold.

2010 Mathematics Subject Classification: 53C15; 53C25; 53B05.

\section{INTRODUCTION}

Friedman and Schouten (1924) first studied semisymmetric metric connection on a differentiable manifold. Hayden (1932) initiated the notion of a metric connection with torsion. Semi-symmetric metric connections were also investigated by Prvanovic (1975), Amur and Pujar (1978), Binh (1990), De and Biswas (1997) and many other geometers.

A semi-symmetric connection in a Riemmanian manifold is defined by Friedman and Schouten (1924) as a connection $\nabla$ whose torsion tensor $T$ satisfies

$$
T\left(X_{1}, X_{2}\right)=\eta\left(X_{2}\right) X_{1}-\eta\left(X_{1}\right) X_{2},
$$

where $g\left(X_{1}, \xi\right)=\eta\left(X_{1}\right)$ is a 1 -form and $\xi$ is a $(1,1)$ tensor field for all vector fields $X_{1}, X_{2} \in \chi\left(M^{n}\right)$ where $\chi\left(M^{n}\right)$ is the set of all differentiable vector fields in $M^{n}$. In addition, if $\nabla g=0$, then $\nabla$ is known as a semi-symmetric metric connection.

The notion of a Kenmotsu manifold was first introduced by Kenmotsu (1972). A Kenmotsu manifold is not Sasakian. Weakly symmetric and weakly Ricci symmetric manifolds were first studied by Tamassy and Binh (1992). De and Ghosh (2005) defined the weakly concircular Ricci symmetric manifolds. Shaikh and Hui (2009) initiated the notion of weakly concircular symmetric manifolds.
A non-flat Riemannian manifold $M^{n}(n>2)$ is called a weakly symmetric manifold (Tamassy and Binh 1992) if $\exists 1$-forms $\mathrm{B}_{1}, B_{2}, B_{3}, B_{4}$ not simultaneously zero such that the curvature tensor $R$ satisfies

$\left(\nabla_{\mathrm{X}_{1}} R\right)\left(X_{2}, X_{3}\right) X_{4}=B_{1}\left(X_{1}\right) R\left(X_{2}, X_{3}\right) X_{4}+B_{2}\left(X_{2}\right) R\left(X_{1}, X_{3}\right) X_{4}+$ $B_{3}\left(X_{3}\right) R\left(X_{2}, X_{1}\right) X_{4}+B_{4}\left(X_{4}\right) R\left(X_{2}, X_{3}\right) X_{1}+g\left(R\left(X_{2}, X_{3}\right) X_{4}, X_{1}\right) P$,

for all $X_{1}, X_{2}, X_{3}, X_{4} \in \chi\left(M^{n}\right)$ and $\nabla$ is the operator of covariant differentiation with respect to the Riemannian metric $g$.

A Riemannian manifold $M^{n}(n>2)$ is known as a weakly Ricci symmetric manifold (Tamassy and Binh 1992) if $\exists 1$-forms $A_{1}, A_{2}, A_{3}$, not simultaneously zero such that the Ricci tensor $S$ is not identically zero and satisfies

$\left(\nabla_{X_{1}} S\right)\left(X_{2}, X_{3}\right)=A_{1}\left(X_{1}\right) S\left(X_{2}, X_{3}\right)+A_{2}\left(X_{2}\right) S\left(X_{1}, X_{3}\right)+$

$A_{3}\left(X_{3}\right) S\left(X_{2}, X_{1}\right)$,

for all $X_{1}, X_{2}, X_{3} \in X\left(M^{n}\right)$.

A Riemannian manifold $M^{n}(n>2)$ is said to be a weakly concircular symmetric manifold (Shaikh and Hui 2009) if $\exists$ $\exists 1$-forms $\mathrm{B}_{1}, B_{2}, B_{3}, B_{4}, B_{5}$ such that

$\left(\nabla_{\mathrm{X}_{1}} C\right)\left(X_{2}, X_{3}, X_{4}, X_{5}\right)=B_{1}\left(X_{1}\right) C\left(X_{2}, X_{3}, X_{4}, X_{5}\right)+$ $B_{2}\left(X_{2}\right) C\left(X_{1}, X_{3}, X_{4}, X_{5}\right)+B_{3}\left(X_{3}\right) C\left(X_{2}, X_{1}, X_{4}, X_{5}\right)+$ $B_{4}\left(X_{4}\right) C\left(X_{2}, X_{3}, X_{1}, X_{5}\right)+B_{5}\left(X_{5}\right) C\left(X_{2}, X_{3}, X_{4}, X_{1}\right)$, 
where $B_{1}, B_{2}, B_{3}, B_{4}, B_{5}$ are not simultaneously zero and the concircular curvature tensor $C$ is given by

$$
\begin{aligned}
C\left(X_{2}, X_{3}, X_{4}, X_{5}\right) & =R\left(X_{2}, X_{3}, X_{4}, X_{5}\right)-\frac{r}{n(n-1)}\left[g\left(X_{3}, X_{4}\right) g\left(X_{2}, X_{5}\right)\right. \\
& \left.-g\left(X_{2}, X_{4}\right) g\left(X_{3}, X_{5}\right)\right]
\end{aligned}
$$

is not identically zero, $r$ being the scalar curvature of the manifold.

A Riemannian manifold $M^{n}(n>2)$ is called a weakly concircular Ricci symmetric manifold (De and Ghosh 2005) if $\exists 1$-forms $A_{1}, A_{2}, A_{3}$, not simultaneously zero such that the concircular Ricci tensor $P$ given by

$P\left(X_{1}, X_{2}\right)=\sum_{i=1}^{n} C\left(E_{i}, X_{1}, X_{2}, E_{i}\right)=S\left(X_{1}, X_{2}\right)-\frac{r}{n} g\left(X_{1}, X_{2}\right)$,

is not identically zero and satisfies
$\left(\nabla_{\mathrm{X}_{1}} P\right)\left(X_{2}, X_{3}\right)=A_{1}\left(X_{1}\right) P\left(X_{2}, X_{3}\right)+A_{2}\left(X_{2}\right) P\left(X_{1}, X_{3}\right)+$

$A_{3}\left(X_{3}\right) P\left(X_{2}, X_{1}\right)$,

for all $X_{1}, X_{2}, X_{3} \in \chi\left(M^{n}\right)$.

The $m$-projective curvature tensor is given by Pokhariyal and Mishra (1971) as

$$
\begin{aligned}
M\left(X_{1}, X_{2}\right) X_{3}= & R\left(X_{1}, X_{2}\right) X_{3}-\frac{1}{2(n-1)}\left[S\left(X_{2}, X_{3}\right) X_{1}-S\left(X_{1}, X_{3}\right) X_{2}\right. \\
& \left.+g\left(X_{2}, X_{3}\right) Q X_{1}-g\left(X_{1}, X_{3}\right) Q X_{2}\right]
\end{aligned}
$$

where $S\left(X_{1}, X_{2}\right)=g\left(Q X_{1}, X_{2}\right)$ is the Ricci tensor of type $(0,2)$ and $Q$ is the Ricci operator.

Since the properties of the Riemannian curvature tensor are also the properties of the $m$-projective curvature tensor, we can define a weakly $m$-projectively symmetric manifold as a non $m$-projectively flat manifold $M^{n}(n>2)$ where

$$
\begin{aligned}
\left(\nabla_{\mathrm{X}_{1}} M\right)\left(X_{2}, X_{3}, X_{4}, X_{5}\right) & =A_{1}\left(X_{1}\right) M\left(X_{2}, X_{3}, X_{4}, X_{5}\right)+A_{2}\left(X_{2}\right) M\left(X_{1}, X_{3}, X_{4}, X_{5}\right) \\
& +A_{3}\left(X_{3}\right) M\left(X_{2}, X_{1}, X_{4}, X_{5}\right)+A_{4}\left(X_{4}\right) M\left(X_{2}, X_{3}, X_{1}, X_{5}\right) \\
& +A_{5}\left(X_{5}\right) M\left(X_{2}, X_{3}, X_{4}, X_{1}\right)
\end{aligned}
$$

where $M\left(X_{2}, X_{3} X_{4}, X_{5}\right)=g\left(M\left(X_{2}, X_{3}\right) X_{4}, X_{5}\right)$ is the associative $m$-projective curvature tensor for all vector fields $X_{1}, X_{2}, X_{3}$, $X_{4}, X_{5} \in X\left(M^{n}\right)$.

In 2015, Prakasha and Vikas studied weakly symmetric properties of Kenmotsu manifolds admitting a quartersymmetric metric connection. This motivated us to study the properties of these manifolds with respect to a semisymmetric metric connection.

This paper is divided into sections as follows: Section 2 is on a brief study of Kenmotsu manifolds. In section 3, we have some basic results on Kenmotsu manifolds admitting a semi-symmetric metric connection. Sections 4 and 5 are devoted to the study of weakly symmetric and weakly Ricci symmetric Kenmotsu manifolds admitting a semisymmetric metric connection. In section 6, some results on weakly concircular and weakly concircular Ricci symmetric Kenmotsu manifolds with respect to a semi-symmetric metric connection are obtained. In section 7 , we study some properties of a weakly $m$-projectively symmetric Kenmotsu manifold admitting a semi-symmetric metric connection. Finally, we construct an example of a weakly symmetric Kenmotsu manifolds with respect to a semi-symmetric metric connection which is also weakly Ricci symmetric.

\section{PRELIMINARIES}

An $n(=2 m+1)$ - dimensional differentiable manifold $M^{n}(n>2)$ is called an almost contact manifold (Blair 1976) if $\exists$ an almost contact structure $(\varphi, \xi, \eta, g)$ satisfying or equivalently,

$\eta(\xi)=1, \quad \varphi(\xi)=0, \quad \eta\left(\varphi X_{1}\right)=0$,

$g\left(\varphi X_{1}, \varphi X_{2}\right)=g\left(X_{1}, X_{2}\right)-\eta\left(X_{1}\right) \eta\left(X_{2}\right)$,

or equivalently,

$g\left(X_{1}, \varphi X_{2}\right)=-g\left(\varphi X_{1}, X_{2}\right), \quad g\left(X_{1}, \xi\right)=\eta\left(X_{1}\right)$,

where $\varphi$ is a $(1,1)$ tensor field, $\xi$ is a vector field, $\eta$ is a 1 -form and $g$ is the Riemmanian metric. In addition, if

$\nabla_{\mathrm{X}_{1}} \xi=X_{1}-\eta\left(X_{1}\right) \xi$,

$\left(\nabla_{X_{1}} \varphi\right)\left(X_{2}\right)=g\left(\varphi X_{1}, X_{2}\right) \xi-\eta\left(X_{2}\right)$,

$\left(\nabla_{X_{1}} \eta\right)\left(X_{2}\right)=g\left(X_{1}, X_{2}\right)-\eta\left(X_{1}\right) \eta\left(X_{2}\right)$,

where, $X_{1}, X_{2}, X_{3} \in X\left(M^{n}\right)$ then $M^{n}$ is called a Kenmotsu manifold. Here, $\nabla$ is the operator of covariant differentiation with respect to the metric $g$.

In a Kenmotsu manifold, we have the following relations (Kenmotsu 1972): 


$$
\begin{aligned}
& R\left(X_{1}, X_{2}\right) \xi=\eta\left(X_{1}\right) X_{2}-\eta\left(X_{2}\right) \xi, \\
& R\left(X_{1}, \xi\right) X_{2}=g\left(X_{1}, X_{2}\right) \xi-\eta\left(X_{2}\right) X_{1}, \\
& R\left(\xi, X_{1}\right) \xi=X_{1}-\eta\left(X_{1}\right) \xi, \\
& S\left(X_{1}, \xi\right)=(n-1) \eta\left(X_{1}\right), \\
& S(\xi, \xi)=(n-1),
\end{aligned}
$$

where $X_{1}, X_{2} \in X\left(M^{n}\right)$ and $R$ and $S$ are the Riemmanian curvature tensor and the Ricci tensor of $M^{n}$ respectively.

\section{SEMI-SYMMETRIC METRIC CONNECTION IN A KENMOTSU MANIFOLD}

A semi-symmetric metric connection in a Kenmotsu manifold is given by Yano (1970) as

$\widetilde{\nabla}_{X_{1}} X_{2}=\nabla_{X_{1}} X_{2}+\eta\left(X_{2}\right) X_{1}-g\left(X_{1}, X_{2}\right) \xi$.

A relation between the Riemmanian curvature tensor $R$ with respect to the Levi-Civita connection $\nabla$ and the curvature tensor $\tilde{R}$ with respect to the semi-symmetric metric connection $\widetilde{\nabla}$ is obtained as (Prakasha et al. 2013)

$$
\begin{aligned}
\tilde{R}\left(X_{1}, X_{2}\right) X_{3}= & R\left(X_{1}, X_{2}\right) X_{3}-3\left[g\left(X_{2}, X_{3}\right) X_{1}-g\left(X_{1}, X_{3}\right) X_{2}\right] \\
& +2\left[\eta\left(X_{2}\right) X_{1}-\eta\left(X_{1}\right) X_{2}\right] \eta\left(X_{3}\right)-2\left[g\left(X_{1}, X_{3}\right) \eta\left(X_{2}\right)\right. \\
& \left.-g\left(X_{2}, X_{3}\right) \eta\left(X_{1}\right)\right] \xi .
\end{aligned}
$$

On contracting equation (3.2), we obtain

$\tilde{S}\left(X_{1}, X_{2}\right)=S\left(X_{1}, X_{2}\right)-(3 n-5) g\left(X_{1}, X_{2}\right)+2(n-2) \eta\left(X_{1}\right) \eta\left(X_{2}\right)$,

where $\tilde{S}$ and $S$ are the Ricci tensors with respect to the semi-symmetric metric connection $\widetilde{\nabla}$ and the Levi-Civita connection $\nabla$ respectively. Again by contraction, (3.3) reduces to

$\tilde{r}=r-2(n-1)$,

where $\tilde{r}$ and $r$ are the scalar curvatures with respect to $\widetilde{\nabla}$ and $\widetilde{\nabla}$ respectively.

By making use of equations (2.2), (2.7), (2.8), (2.9), (2.10) and (2.11), we obtain

$$
\begin{aligned}
& \tilde{R}\left(X_{1}, X_{2}\right) \xi=2\left[\eta\left(X_{1}\right) X_{2}-\eta\left(X_{2}\right) \xi\right], \\
& \tilde{R}\left(X_{1}, \xi\right) X_{2}=2\left[g\left(X_{1}, X_{2}\right) \xi-\eta\left(X_{2}\right) X_{1}\right], \\
& \tilde{R}\left(\xi, X_{1}\right) \xi=2\left[X_{1}-\eta\left(X_{1}\right) \xi\right], \\
& \tilde{S}\left(X_{1}, \xi\right)=2\left[(n-1) \eta\left(X_{1}\right)\right], \\
& \tilde{S}(\xi, \xi)=2(n-1) .
\end{aligned}
$$

\section{WEAKLY SYMMETRIC KENMOTSU MANIFOLDS ADMITTING A SEMI- SYMMETRIC METRIC CONNECTION}

Definition 4.1. A non-flat Kenmotsu manifold $M^{n}(n>2)$ is called a weakly symmetric Kenmotsu manifold with respect to the semi-symmetric metric connection $\widetilde{\nabla}$ if $\exists 1$-forms $B_{1}, B_{2}, B_{3}, B_{4}$, not simultaneously zero and the curvature tensor $\tilde{R}$ with respect to the semi-symmetric metric connection $\widetilde{\nabla}$ satisfies

$$
\begin{aligned}
\left(\widetilde{\nabla}_{\mathrm{X}_{1}} \tilde{R}\right)\left(X_{2}, X_{3}\right) X_{4}= & B_{1}\left(X_{1}\right) \tilde{R}\left(X_{2}, X_{3}\right) X_{4}+B_{2}\left(X_{2}\right) \tilde{R}\left(X_{1}, X_{3}\right) X_{4} \\
& +B_{3}\left(X_{3}\right) \tilde{R}\left(X_{2}, X_{1}\right) X_{4}+B_{4}\left(X_{4}\right) \tilde{R}\left(X_{2}, X_{3}\right) X_{1} \\
& +g\left(\tilde{R}\left(X_{2}, X_{3}\right) X_{4}, X_{1}\right) P
\end{aligned}
$$

for all vector fields $X_{1}, X_{2}, X_{3}, X_{4} \in X\left(M^{n}\right)$.

Theorem 4.1: In a weakly symmetric Kenmotsu manifold with respect to a semi-symmetric metric connection $\widetilde{\nabla}$, the sum of the 1 -forms $B_{1}+B_{3}+B_{4}$ vanishes.

Proof: Suppose equation (4.1) holds. Then, by contracting (4.1) with respect to, $X_{2}$ we obtain

$\left(\widetilde{\nabla}_{\mathrm{X}_{1}} \tilde{S}\right)\left(X_{3}, X_{4}\right)=B_{1}\left(X_{1}\right) \tilde{S}\left(X_{3}, X_{4}\right)+B_{2}\left(\tilde{R}\left(X_{1}, X_{3}\right) X_{4}\right)$

$+B_{3}\left(X_{3}\right) \tilde{S}\left(X_{1}, X_{4}\right)+B_{4}\left(X_{4}\right) \tilde{S}\left(X_{3}, X_{1}\right)+B\left(\tilde{R}\left(X_{1}, X_{4}\right) X_{3}\right)$,

where $B\left(X_{1}\right)=g\left(X_{1}, P\right)$. Replacing $X_{4}$ by $\xi$ in equation (4.2), we have

$$
\begin{array}{r}
\left(\widetilde{\nabla}_{\mathrm{X}_{1}} \tilde{S}\right)\left(X_{3}, \xi\right)=B_{1}\left(X_{1}\right) \tilde{S}\left(X_{3}, \xi\right)+B_{2}\left(\tilde{R}\left(X_{1}, X_{3}\right) \xi\right) \\
+B_{3}\left(X_{3}\right) \tilde{S}\left(X_{1}, \xi\right)+B_{4}(\xi) \tilde{S}\left(X_{3}, X_{1}\right)+B\left(\tilde{R}\left(X_{1}, \xi\right) X_{3}\right) .
\end{array}
$$

Applying equations (3.5), (3.7), (3.8) in (4.3), we get

$$
\begin{aligned}
\left(\widetilde{\nabla}_{\mathrm{X}_{1}} \tilde{S}\right)\left(X_{3}, \xi\right) & =-2(n-1)\left[B_{1}\left(X_{1}\right) \eta\left(X_{3}\right)+B_{3}\left(X_{3}\right) \eta\left(X_{1}\right)\right] \\
& +2 \eta\left(X_{1}\right) B_{2}\left(X_{3}\right)-2 \eta\left(X_{3}\right)\left[B_{2}\left(X_{1}\right)+B\left(X_{1}\right)\right] \\
& +B_{4}(\xi) \tilde{S}\left(X_{1}, X_{3}\right)+2 B(\xi) g\left(X_{1}, X_{3}\right) .
\end{aligned}
$$

Also,

$\left(\widetilde{\nabla}_{\mathrm{X}_{1}} \tilde{S}\right)\left(X_{3}, \xi\right)=\widetilde{\nabla}_{\mathrm{X}_{1}} \tilde{S}\left(X_{3}, \xi\right)-\tilde{S}\left(X_{3}, \widetilde{\nabla}_{\mathrm{X}_{1}} \xi\right)-\tilde{S}\left(\widetilde{\nabla}_{\mathrm{X}_{1}} X_{3}, \xi\right)$.

Using (2.3), (3.1) in (4.5), we get

$\left(\widetilde{\nabla}_{\mathrm{X}_{1}} \tilde{S}\right)\left(X_{3}, \xi\right)=-4(n-1)\left[g\left(X_{1}, X_{3}\right)-\right.$

$\left.\eta\left(X_{1}\right) \eta\left(X_{3}\right)\right]-2 \tilde{S}\left(X_{1}, X_{3}\right)-\eta\left(X_{1}\right) \xi$.

Comparing (4.4) and (4.6), we obtain 


$$
\begin{aligned}
-4(n-1)[ & \left.g\left(X_{1}, X_{3}\right)-\eta\left(X_{1}\right) \eta\left(X_{3}\right)\right]-2 \tilde{S}\left(X_{1}, X_{3}\right)-\eta\left(X_{1}\right) \xi \\
& =-2(n-1)\left[B_{1}\left(X_{1}\right) \eta\left(X_{3}\right)+B_{3}\left(X_{3}\right) \eta\left(X_{1}\right)\right] \\
& +2 \eta\left(X_{1}\right) B_{2}\left(X_{3}\right)-2 \eta\left(X_{3}\right)\left[B_{2}\left(X_{1}\right)+B\left(X_{1}\right)\right] \\
& +B_{4}(\xi) \tilde{S}\left(X_{1}, X_{3}\right)+2 B(\xi) g\left(X_{1}, X_{3}\right) .
\end{aligned}
$$

Taking $X_{1}=X_{3}=\xi$,in (4.7), we have

$-2(n-1)\left[B_{1}(\xi)+B_{3}(\xi)+B_{4}(\xi)\right]=0$.

Since, this implies

$B_{1}(\xi)+B_{3}(\xi)+B_{4}(\xi)=0$.

In (4.2), we take $X_{3}=\xi$ and get,

$\left(\widetilde{\nabla}_{\mathrm{X}_{1}} \tilde{S}\right)\left(\xi, X_{4}\right)=-2(n-1)\left[B_{1}\left(X_{1}\right) \eta\left(X_{4}\right)+B\left(X_{4}\right) \eta\left(X_{1}\right)\right]+2 B_{2}(\xi) g\left(X_{1}, X_{4}\right)$ $-2 \eta\left(X_{4}\right)\left[B_{2}\left(X_{1}\right)+B\left(X_{1}\right)\right]+2 B\left(X_{4}\right) \eta\left(X_{1}\right)+B_{3}(\xi)$.

Also,

$\left(\widetilde{\nabla}_{\mathrm{X}_{1}} \tilde{S}\right)\left(\xi, X_{4}\right)=-4(n-1)\left[g\left(X_{1}, X_{4}\right)-\eta\left(X_{1}\right) \eta\left(X_{4}\right)\right]-2 \tilde{S}\left(X_{1}-\eta\left(X_{1}\right) \xi, X_{4}\right)$.

Using (4.9) in (4.10) we obtain,

$$
\begin{gathered}
-4(n-1)\left[g\left(X_{1}, X_{4}\right)-\eta\left(X_{1}\right) \eta\left(X_{4}\right)\right]-2 \tilde{S}\left(X_{1}-\eta\left(X_{1}\right) \xi, X_{4}\right) \\
=-2(n-1)\left[B_{1}\left(X_{1}\right) \eta\left(X_{4}\right)+B\left(X_{4}\right) \eta\left(X_{1}\right)\right]+2 B_{2}(\xi) g\left(X_{1}, X_{4}\right) \\
-2 \eta\left(X_{4}\right)\left[B_{2}\left(X_{1}\right)+B\left(X_{1}\right)\right]+2 B\left(X_{4}\right) \eta\left(X_{1}\right)+B_{3}(\xi) . \\
\text { Setting } X_{4}=\xi \operatorname{in}(4.11), \text { we get } \\
-2(n-1)\left[B_{1}\left(X_{1}\right)+B_{3}(\xi) \eta\left(X_{1}\right)+B_{4}(\xi) \eta\left(X_{1}\right)\right]+2 B_{2}(\xi) \eta\left(X_{1}\right) \\
+2 B(\xi) \eta\left(X_{1}\right)-2\left[B_{2}\left(X_{1}\right)+B\left(X_{1}\right)\right]=0 .
\end{gathered}
$$

Take $X_{1}=\xi$ in (4.11), we obtain

$$
\begin{aligned}
-2(n-1)\left[B_{1}(\xi) \eta\left(X_{4}\right)+\right. & \left.B_{4}\left(X_{4}\right)+B_{3}(\xi) \eta\left(X_{4}\right)\right] \\
& -2 \eta\left(X_{4}\right) B(\xi)+2 B\left(X_{4}\right)=0 .
\end{aligned}
$$

for all $X_{1}, X_{2}, X_{3} \in \chi\left(M^{n}\right)$.

Theorem 5.1. The necessary condition for a Kenmotsu manifold with respect to the semi-symmetric metric connection $\widetilde{\nabla}$ to be weakly Ricci symmetric with respect to $\widetilde{\nabla}$ is that the sum of the associated 1-forms $A_{1}, A_{2}, A_{3}$ vanishes everywhere.

Proof: On replacing $X_{3}$ by $\xi$ in (5.1), we obtain

$$
\begin{aligned}
\left(\widetilde{\nabla}_{\mathrm{X}_{1}} \tilde{S}\right)\left(X_{2}, \xi\right)=A_{1}\left(X_{1}\right) \tilde{S}\left(X_{2}, \xi\right) & +A_{2}\left(X_{2}\right) \tilde{S}\left(X_{1}, \xi\right) \\
& +A_{3}(\xi) \tilde{S}\left(X_{2}, X_{1}\right),
\end{aligned}
$$

which implies on using equation (4.7),

$$
\begin{aligned}
& -4(n-1)\left[g\left(X_{1}, X_{2}\right)-\eta\left(X_{1}\right) \eta\left(X_{2}\right)\right]-2 \tilde{S}\left(X_{2}, X_{1}-\eta\left(X_{1}\right) \xi\right) \\
& =-2(n-1)\left[A_{1}\left(X_{1}\right) \eta\left(X_{2}\right)+A_{2}\left(X_{2}\right) \eta\left(X_{1}\right)\right]+A_{3}(\xi) \tilde{S}\left(X_{1}, X_{2}\right) .
\end{aligned}
$$

In (4.10), taking $X_{1}=X_{2}=\xi$ we get

$A_{1}(\xi)+A_{2}(\xi)+A_{3}(\xi)=0$.

Now, substituting $X_{2}=\xi$ equation (5.4) becomes

$A_{1}\left(X_{1}\right)=A_{1}(\xi) \eta\left(X_{1}\right)$. 


\section{Some Results on Weakly Symmetric Kenmotsu Manifolds}

Similarly, we can obtain

$A_{2}\left(X_{2}\right)=A_{2}(\xi) \eta\left(X_{2}\right)$

and

$A_{3}\left(X_{3}\right)=A_{3}(\xi) \eta\left(X_{3}\right)$.

Taking the sum of (5.5), (5.6) and (5.7), we obtain

$A_{1}\left(X_{1}\right)+A_{2}\left(X_{1}\right)+A_{3}\left(X_{1}\right)=0$.

This proves the theorem.

\section{WEAKLY CONCIRCULAR SYMMETRIC AND WEAKLY CONCIRCULAR RICCI KENMOTSU MANIFOLDS ADMITTING A SEMI- SYMMETRIC CONNECTION}

Definition 6.1. A Kenmotsu manifold $M^{n}(n>2)$ is called a weakly concircular symmetric Kenmotsu manifold with respect to the semi-symmetric metric connection $\widetilde{\nabla}$ if $\exists$ 1-forms (not simultaneously zero) $B_{1}, B_{2}, B_{3}, B_{4}, B_{5}$ such that $\left(\widetilde{\nabla}_{\mathrm{X}_{1}} \tilde{C}\right)\left(X_{2}, X_{3}, X_{4}, X_{5}\right)=B_{1}\left(X_{1}\right) \tilde{C}\left(X_{2}, X_{3}, X_{4}, X_{5}\right)$

$+B_{2}\left(X_{2}\right) \tilde{C}\left(X_{1}, X_{3}, X_{4}, X_{5}\right)+B_{3}\left(X_{3}\right) \tilde{C}\left(X_{2}, X_{1}, X_{4}, X_{5}\right)$

$+B_{4}\left(X_{4}\right) \tilde{C}\left(X_{2}, X_{3}, X_{1}, X_{5}\right)+B_{5}\left(X_{5}\right) \tilde{C}\left(X_{2}, X_{3}, X_{4}, X_{1}\right)$,

where

$$
\begin{array}{r}
\tilde{C}\left(X_{2}, X_{3}, X_{4}, X_{5}\right)=\tilde{R}\left(X_{2}, X_{3}, X_{4}, X_{5}\right)-\frac{\tilde{r}}{n(n-1)}\left[g\left(X_{3}, X_{4}\right) g\left(X_{2}, X_{5}\right)\right. \\
\left.-g\left(X_{2}, X_{4}\right) g\left(X_{3}, X_{5}\right)\right]
\end{array}
$$

is the concircular curvature tensor which is not identically zero.

In a weakly concircular symmetric Kenmotsu manifold admittingasemi-symmetricmetricconnection, $B_{2}=B_{3}$ and $B_{4}=B_{5}$. So (6.1) can be written as

$\left(\widetilde{\nabla}_{\mathrm{X}_{1}} \tilde{C}\right)\left(X_{2}, X_{3}, X_{4}, X_{5}\right)=B_{1}\left(X_{1}\right) \tilde{C}\left(X_{2}, X_{3}, X_{4}, X_{5}\right)$

$+B_{2}\left(X_{2}\right) \tilde{C}\left(X_{1}, X_{3}, X_{4}, X_{5}\right)+B_{2}\left(X_{3}\right) \tilde{C}\left(X_{2}, X_{1}, X_{4}, X_{5}\right)$

$+B_{4}\left(X_{4}\right) \tilde{C}\left(X_{2}, X_{3}, X_{1}, X_{5}\right)+B_{4}\left(X_{5}\right) \tilde{C}\left(X_{2}, X_{3}, X_{4}, X_{1}\right)$.

Substituting $X_{2}=X_{5}=E_{i}$ in (6.2) and taking summation over $i, 1 \leq i \leq n$, we get

$$
\begin{aligned}
\left(\widetilde{\nabla}_{X_{1}} \tilde{S}\right)\left(X_{3}, X_{4}\right)-\frac{d \tilde{r}}{n} g\left(X_{3}, X_{4}\right) & =B_{1}\left(X_{1}\right)\left[\tilde{S}\left(X_{3}, X_{4}\right)-\frac{\tilde{r}}{n} g\left(X_{3}, X_{4}\right)\right] \\
& +B_{2}\left(X_{3}\right)\left[\tilde{S}\left(X_{1}, X_{4}\right)-\frac{\tilde{r}}{n} g\left(X_{1}, X_{4}\right)\right] \\
& +B_{4}\left(X_{4}\right)\left[\tilde{S}\left(X_{3}, X_{1}\right)-\frac{\tilde{r}}{n} g\left(X_{3}, X_{1}\right)\right] \\
& +B_{2}\left(\tilde{R}\left(X_{1}, X_{3}\right) X_{4}\right)+B_{4}\left(\tilde{R}\left(X_{1}, X_{4}\right) X_{3}\right) \\
& -\frac{\tilde{r}}{n(n-1)}\left[g\left(X_{3}, X_{4}\right)\left\{B_{2}\left(X_{1}\right)+B_{4}\left(X_{1}\right)\right\}\right.
\end{aligned}
$$

$\left.-B_{2}\left(X_{3}\right) g\left(X_{1}, X_{4}\right)-B_{4}\left(X_{4}\right) g\left(X_{1}, X_{3}\right)\right]$.

Taking $X_{1}=X_{3}=X_{4}=\xi$,equation (6.3) reduces to

$B_{1}(\xi)+B_{2}(\xi)+B_{4}(\xi)=\frac{d r}{\tilde{r}+2 n(n-1)}$,

provided $\tilde{r}+2 n(n-1) \neq 0$.

Substituting $X_{1}, X_{3}$ by $\xi$ in (6.3) and using (6.4), we have

$B_{4}\left(X_{4}\right)=B_{4}(\xi) \eta\left(X_{4}\right)$.

Similarly, on substituting $X_{1}, X_{4}$ by $\xi$ in (6.3) and using (6.4), we obtain

$B_{2}\left(X_{3}\right)=B_{2}(\xi) \eta\left(X_{3}\right)$,

and taking $X_{3}=X_{4}=\xi$,in (57) and using equations (3.5), (6.4), (6.5) and (6.6) we get

$B_{1}\left(X_{1}\right)=\frac{d \tilde{r}}{\tilde{r}+2 n(n-1)}\left[B_{2}(\xi)+B_{4}(\xi)\right] \eta\left(X_{1}\right)$.

This leads to the following theorem:

Theorem 6.1: In a weakly concircular symmetric Kenmotsu manifold admitting a semi-symmetric metric connection $\widetilde{\nabla}$, the sum of the associated 1-forms $B_{1}, B_{2}, B_{4}$, is given by (6.7).

Definition 6.2: A Kenmotsu manifold $M^{n}(n>2)$ is called a weakly concircular Ricci symmetric manifold with respect to the semi-symmetric metric connection $\widetilde{\nabla}$ if $\exists 1$-forms $A_{1}, A_{2}, A_{3}$, not simultaneously zero such that the concircular Ricci tensor $\tilde{P}$ with respect to $\widetilde{\nabla}$ given by

$\tilde{P}\left(X_{1}, X_{2}\right)=\sum_{i=1}^{n} \tilde{C}\left(E_{i}, X_{1}, X_{2}, E_{i}\right)=\tilde{S}\left(X_{1}, X_{2}\right)-\frac{r}{n} g\left(X_{1}, X_{2}\right)$

is not identically zero and satisfies

$$
\begin{aligned}
\left(\widetilde{\nabla}_{\mathrm{X}_{1}} \tilde{P}\right)\left(X_{2}, X_{3}\right)=A_{1}\left(X_{1}\right) \tilde{P}\left(X_{2}, X_{3}\right) & +A_{2}\left(X_{2}\right) \tilde{P}\left(X_{1}, X_{3}\right) \\
& +A_{3}\left(X_{3}\right) \tilde{P}\left(X_{2}, X_{1}\right)
\end{aligned}
$$

for all $X_{1}, X_{2}, X_{3} \in \chi\left(M^{n}\right)$.

Suppose (6.8) holds. Then, we have

$$
\begin{aligned}
\left(\widetilde{\nabla}_{X_{1}} \tilde{S}\right)\left(X_{2}, X_{3}\right)-\frac{d \tilde{r}\left(X_{1}\right)}{n} & g\left(X_{2}, X_{3}\right) \\
= & A_{1}\left(X_{1}\right)\left[\tilde{S}\left(X_{2}, X_{3}\right)-\frac{\tilde{r}}{n} g\left(X_{2}, X_{3}\right)\right] \\
& +A_{2}\left(X_{2}\right)\left[\tilde{S}\left(X_{1}, X_{3}\right)-\frac{\tilde{r}}{n} g\left(X_{1}, X_{3}\right)\right] \\
& \quad+A_{3}\left(X_{3}\right)\left[\tilde{S}\left(X_{2}, X_{1}\right)-\frac{\tilde{r}}{n} g\left(X_{2}, X_{1}\right)\right] .(6.9)
\end{aligned}
$$

On replacing $X_{1}, X_{2}, X_{3}$ by $\xi$,in (6.9), we obtain 
$A_{1}(\xi)+A_{2}(\xi)+A_{3}(\xi)=\frac{d \tilde{r}(\xi)}{\tilde{r}+2 n(n-1)}$

provided $\tilde{r}+2 n(n-1) \neq 0$.

Taking $X_{1}=X_{2}=\xi$ in (6.9) and using (6.8), we get

$$
A_{3}\left(X_{3}\right)=A_{3}(\xi) \eta\left(X_{3}\right) \text {. }
$$

Similarly, replacing $X_{1}, X_{3}$ by $\xi$,and using (6.10), (6.9) becomes $A_{2}\left(X_{2}\right)=A_{2}(\xi) \eta\left(X_{2}\right)$

and replacing $X_{2}, X_{3}$ by $\xi$,in (6.9) and using (6.10), we get

$A_{1}\left(X_{1}\right)=\frac{d \tilde{r}\left(X_{1}\right)}{\tilde{r}+2 n(n-1)}+\left[A_{1}(\xi)-\frac{d \tilde{r}(\xi)}{\tilde{r}+2 n(n-1)}\right] \eta\left(X_{1}\right)$

provided $\tilde{r}+2 n(n-1) \neq 0$.

Adding equations (6.11), (6.12) and (6.13), we have

$A_{1}\left(X_{1}\right)+A_{2}\left(X_{1}\right)+A_{3}\left(X_{1}\right)=\frac{d \tilde{r}\left(X_{1}\right)}{\tilde{r}+2 n(n-1)}$,

provided $\tilde{r}+2 n(n-1) \neq 0$. This leads to the following theorem:

Theorem 6.2: The sum of the associated 1-forms $A_{1}, A_{2}, A_{3}$ in a weakly concircular Ricci symmetric Kenmotsu manifold which admits a semi-symmetric metric connection $\widetilde{\nabla}$ is given by (6.14).

\section{WEAKLY $m$-PROJECTIVELY SYMMETRIC KENMOTSU MANIFOLDS ADMITTING A SEMI-SYMMETRIC METRIC CONNECTION}

Definition 7.1: A non $m$-projectively flat Kenmotsu manifold $M^{n}(n>2)$ is said to be weakly $m$-projectively flat with respect to the semi-symmetric metric connection $\widetilde{\nabla}$ if the $m$-projective curvature tensor $\widetilde{M}$ with respect to the connection $\widetilde{\nabla}$ given by

$$
\begin{aligned}
\widetilde{M}\left(X_{1}, X_{2}\right) X_{3} & =\tilde{R}\left(X_{1}, X_{2}\right) X_{3}-\frac{1}{2(n-1)}\left[\tilde{S}\left(X_{2}, X_{3}\right) X_{1}-\tilde{S}\left(X_{1}, X_{3}\right) X_{2}\right. \\
& \left.+g\left(X_{2}, X_{3}\right) \tilde{Q} X_{1}-g\left(X_{1}, X_{3}\right) \tilde{Q} X_{2}\right]
\end{aligned}
$$

is not identically zero and satisfies

$$
\begin{array}{r}
\left(\widetilde{\nabla}_{X_{1}} \tilde{M}\right)\left(X_{2}, X_{3}, X_{4}, X_{5}\right)=A_{1}\left(X_{1}\right) \tilde{M}\left(X_{2}, X_{3}, X_{4}, X_{5}\right) \\
+A_{2}\left(X_{2}\right) \tilde{M}\left(X_{1}, X_{3}, X_{4}, X_{5}\right)+A_{3}\left(X_{3}\right) \tilde{M}\left(X_{2}, X_{1}, X_{4}, X_{5}\right) \\
A_{4}\left(X_{4}\right) \tilde{M}\left(X_{2}, X_{3}, X_{1}, X_{5}\right)+A_{5}\left(X_{5}\right) \tilde{M}\left(X_{2}, X_{3}, X_{4}, X_{1}\right),
\end{array}
$$

for all vector fields $X_{1}, X_{2}, X_{3}, X_{4}, X_{5}, \in \chi\left(M^{n}\right)$ and $A_{1}, A_{2}, A_{3}, A_{4}, A_{5}$ are 1-forms, not simultaneously zero.

In a weakly $m$-projectively symmetric Kenmotsu manifold admitting a semi-symmetric metric connection, $A_{2}=A_{3}, A_{4}=A_{5}$. So (7.2) can be written as

$$
\begin{array}{r}
\left(\widetilde{\nabla}_{X_{1}} \tilde{M}\right)\left(X_{2}, X_{3}, X_{4}, X_{5}\right)=A_{1}\left(X_{1}\right) \tilde{M}\left(X_{2}, X_{3}, X_{4}, X_{5}\right) \\
+A_{2}\left(X_{2}\right) \tilde{M}\left(X_{1}, X_{3}, X_{4}, X_{5}\right)+A_{2}\left(X_{3}\right) \tilde{M}\left(X_{2}, X_{1}, X_{4}, X_{5}\right) \\
+A_{4}\left(X_{4}\right) \tilde{M}\left(X_{2}, X_{3}, X_{1}, X_{5}\right)+A_{4}\left(X_{5}\right) \tilde{M}\left(X_{2}, X_{3}, X_{4}, X_{1}\right) .
\end{array}
$$

From equation (7.1), we can obtain

$$
\begin{aligned}
\sum_{i=1}^{n} \tilde{M}\left(E_{i}, X_{2}, X_{3}, E_{i}\right) & =\frac{n}{2(n-1)}\left[\tilde{S}\left(X_{2}, X_{3}\right)-\frac{\tilde{r}}{n} g\left(X_{2}, X_{3}\right)\right] \\
& =\frac{n}{2(n-1)} \tilde{W}\left(X_{2}, X_{3}\right),
\end{aligned}
$$

where $\widetilde{W}\left(X_{2}, X_{3}\right)=\tilde{S}\left(X_{2}, X_{3}\right)-\frac{\tilde{r}}{n} g\left(X_{2}, X_{3}\right)$.

Also,

$$
\begin{aligned}
& \sum_{i=1}^{n} \widetilde{M}\left(X_{1}, X_{2}, E_{i}, E_{i}\right)=0, \\
& \sum_{i=1}^{n} \widetilde{M}\left(E_{i}, E_{i}, E_{i}, E_{i}\right)=0 .
\end{aligned}
$$

Also, the $m$-projective curvature tensor $\widetilde{M}$ with respect to $\widetilde{\nabla}$ satisfies

$$
\begin{array}{r}
\tilde{M}\left(X_{1}, X_{2}, X_{3}, X_{4}\right)+\tilde{M}\left(X_{2}, X_{3}, X_{1}, X_{4}\right) \\
+\widetilde{M}\left(X_{3}, X_{1}, X_{2}, X_{4}\right)=0, \\
\tilde{M}\left(X_{1}, X_{2}, X_{4}, X_{3}\right)+\tilde{M}\left(X_{2}, X_{3}, X_{4}, X_{1}\right) \\
+\widetilde{M}\left(X_{3}, X_{1}, X_{4}, X_{2}\right)=0 .
\end{array}
$$

\section{NATURE OF THE SCALAR CURVATURE WITH RESPECT TO THE SEMI-SYMMETRIC METRIC CONNECTION}

Let $\tilde{Q}$ be the Ricci operator with respect to the semisymmetric metric connection $\widetilde{\nabla}$ defined by $g\left(\tilde{Q} X_{1}, X_{2}\right)=\tilde{S}\left(X_{1}, X_{2}\right)$.

Differentiating equation (7.7) covariantly along $X_{1}$ and using Bianchi identity, we get 
$\left(\widetilde{\nabla}_{\mathrm{X}_{1}} \widetilde{M}\right)\left(X_{2}, X_{3}, X_{4}, X_{5}\right)+\left(\widetilde{\nabla}_{\mathrm{X}_{2}} \widetilde{M}\right)\left(X_{3}, X_{1}, X_{4}, X_{5}\right)+$

$+\left(\widetilde{\nabla}_{\mathrm{X}_{3}} \widetilde{M}\right)\left(X_{1}, X_{2}, X_{4}, X_{5}\right)$

$=-\frac{1}{2(n-1)}\left[\left\{\left(\widetilde{\nabla}_{X_{1}} \tilde{S}\right)\left(X_{3}, X_{4}\right)-\left(\widetilde{\nabla}_{\mathrm{X}_{3}} S\right)\left(X_{1}, X_{4}\right)\right\} g\left(X_{2}, X_{5}\right)\right.$

$+\left\{\left(\widetilde{\nabla}_{\mathrm{X}_{2}} \tilde{S}\right)\left(X_{1}, X_{4}\right)-\left(\widetilde{\nabla}_{\mathrm{X}_{1}} \tilde{S}\right)\left(X_{2}, X_{4}\right)\right\} g\left(X_{3}, X_{5}\right)$

$+\left\{\left(\widetilde{\nabla}_{\mathrm{X}_{3}} \tilde{S}\right)\left(X_{2}, X_{4}\right)-\left(\widetilde{\nabla}_{\mathrm{X}_{2}} \tilde{S}\right)\left(X_{3}, X_{4}\right)\right\} g\left(X_{1}, X_{5}\right)$

$+\left\{\left(\widetilde{\nabla}_{\mathrm{X}_{1}} \tilde{S}\right)\left(X_{2}, X_{5}\right)-\left(\widetilde{\nabla}_{\mathrm{X}_{2}} \tilde{S}\right)\left(X_{1}, X_{5}\right)\right\} g\left(X_{3}, X_{4}\right)$

$\left.+\left\{\left(\widetilde{\nabla}_{\mathrm{X}_{2}} \tilde{S}\right)\left(X_{3}, X_{5}\right)-\left(\widetilde{\nabla}_{\mathrm{X}_{3}} \tilde{S}\right)\left(X_{2}, X_{5}\right)\right\} g\left(X_{1}, X_{4}\right)\right]$.

Suppose the Ricci tensor $\tilde{S}$ is of Codazzi type, then

$\left(\widetilde{\nabla}_{\mathrm{X}_{1}} \tilde{S}\right)\left(X_{2}, X_{3}\right)=\left(\widetilde{\nabla}_{\mathrm{X}_{2}} \tilde{S}\right)\left(X_{1}, X_{3}\right)$.

Using (7.10) in (7.9), we get

$$
\begin{array}{r}
\left(\widetilde{\nabla}_{\mathrm{X}_{1}} \widetilde{M}\right)\left(X_{2}, X_{3}, X_{4}, X_{5}\right)+\left(\widetilde{\nabla}_{\mathrm{X}_{2}} \widetilde{M}\right)\left(X_{3}, X_{1}, X_{4}, X_{5}\right) \\
+\left(\widetilde{\nabla}_{\mathrm{X}_{3}} \widetilde{M}\right)\left(X_{1}, X_{2}, X_{4}, X_{5}\right)=0 .
\end{array}
$$

Suppose (7.11) holds. Then, clearly the Ricci tensor $\tilde{S}$ is of Codazzi type. This leads to the theorem:

Theorem 7.1: In a weakly $m$-projectively symmetric Kenmotsu manifold, the Ricci tensor $\tilde{S}$ with respect to the semi-symmetric metric connection $\widetilde{\nabla}$ is of Codazzi type if and only if the relation (7.11) holds.

Suppose the Ricci tensor $\tilde{S}$ is of Codazzi type. Then, (7.11) holds. Using (7.2) in (7.11), we get

$$
\begin{array}{r}
\lambda\left(X_{1}\right) \widetilde{M}\left(X_{2}, X_{3}, X_{4}, X_{5}\right)+\lambda\left(X_{2}\right) \widetilde{M}\left(X_{1}, X_{3}, X_{4}, X_{5}\right) \\
+\lambda\left(X_{3}\right) \widetilde{M}\left(X_{2}, X_{1}, X_{4}, X_{5}\right)=0,
\end{array}
$$

where, $\lambda\left(X_{1}\right)=A_{1}\left(X_{1}\right)-2 A_{2}\left(X_{1}\right)$,

for all $X_{1}, X_{2}, X_{3}, X_{4} \in \chi\left(M^{n}\right)$.

Putting $X_{2}=X_{5}=E_{i}$ and taking summation over $i, 1 \leq i \leq n$, (7.12) reduces to

$$
\begin{aligned}
& \frac{n}{2(n-1)}\left[\lambda\left(X_{1}\right) \tilde{S}\left(X_{3}, X_{4}\right)-\frac{\tilde{r}}{n} g\left(X_{3}, X_{4}\right)\right. \\
& \left.-\lambda\left(X_{3}\right) \tilde{S}\left(X_{1}, X_{4}\right)+\frac{\tilde{r}}{n} g\left(X_{1}, X_{4}\right)\right]=0 .
\end{aligned}
$$

Again, substituting $X_{1}=X_{4}=E_{i}$ in (7.12) and summing over $i, 1 \leq i \leq n$, we get $\lambda\left(\tilde{Q} X_{3}\right)=\frac{\tilde{r}}{n} \lambda\left(X_{3}\right)$, which implies that

$\tilde{S}\left(X_{3}, X\right)=\frac{\tilde{r}}{n} g\left(X_{3}, X\right)$.

Thus, we have the theorem:

Theorem 7.2: If the Ricci tensor $\tilde{S}$ in a weakly $m$-projectively symmetric Kenmotsu manifold admitting a semi-symmetric motrir rnnnection $\widetilde{\nabla}$ is of Codazzi type, then $\frac{\tilde{r}}{\eta}$ is an eigen value rresponding to the eigen vector $X$ defined by $g\left(X_{1}, X\right)=\lambda\left(X_{1}\right)$.

Example: Let $M^{3}=\{(x, y, z) \in \mathbb{R}: x \neq 0\}$. Choose linearly independent vectors in $M^{3}$,

$E_{1}=-x \frac{\partial}{\partial x}, \quad E_{2}=x \frac{\partial}{\partial y}, \quad E_{3}=x \frac{\partial}{\partial z}$.

Let $g$ be the Riemannian metric given by

$$
\begin{aligned}
& g\left(E_{1}, E_{1}\right)=g\left(E_{2}, E_{2}\right)=g\left(E_{3}, E_{3}\right)=1, \\
& g\left(E_{1}, E_{2}\right)=g\left(E_{2}, E_{3}\right)=g\left(E_{3}, E_{1}\right)=0 .
\end{aligned}
$$

Let $\varphi\left(E_{1}\right)=0, \varphi\left(E_{2}\right)=E_{3}, \varphi\left(E_{3}\right)=-E_{2}$ be the $(1,1)$ tensor field, and let the 1 -form $\eta$ be given by $\eta\left(X_{3}\right)=g\left(X_{3}, E_{1}\right)$ for any vector field $X_{3} \in \chi\left(M^{3}\right)$. Then, by linearity of $\varphi$ and $g$, we have

$$
\begin{gathered}
\eta\left(E_{1}\right)=1, \quad \varphi^{2} X_{1}=-X_{1}+\eta\left(X_{1}\right), \\
g\left(\varphi X_{1}, \varphi X_{2}\right)=g\left(X_{1}, X_{2}\right)-\eta\left(X_{1}\right) \eta\left(X_{2}\right),
\end{gathered}
$$

for any vector fields $X_{1}, X_{2} \in \chi\left(M^{3}\right)$. So for $\xi=E_{1},(\varphi, \xi, \eta, g)$ is an almost contact structure on $M^{3}$. Let $\nabla$ be the Levi-Civita connection with respect to $g$. Then, we have

$\left[E_{1}, E_{2}\right]=-E_{2}, \quad\left[E_{2}, E_{3}\right]=0, \quad\left[E_{1}, E_{3}\right]=-E_{3}$.

The Riemannian connection $\widetilde{\nabla}$ of the metric $g$ is given by Koszul's formula,

$$
\begin{gathered}
2 g\left(\nabla_{X_{1}} X_{2}, X_{3}\right)=X_{1} g\left(X_{2}, X_{3}\right)+X_{2} g\left(X_{1}, X_{3}\right)-X_{3} g\left(X_{1}, X_{2}\right) \\
-g\left(X_{1},\left[X_{2}, X_{3}\right]\right)-g\left(X_{2},\left[X_{1}, X_{3}\right]\right)+g\left(X_{3},\left[X_{1}, X_{2}\right]\right),
\end{gathered}
$$

which yields

$$
\begin{gathered}
\nabla_{\mathrm{E}_{1}} E_{2}=0, \quad \nabla_{\mathrm{E}_{1}} E_{3}=0, \quad \nabla_{\mathrm{E}_{1}} E_{1}=0, \\
\nabla_{\mathrm{E}_{2}} E_{3}=0, \quad \nabla_{\mathrm{E}_{2}} E_{2}=-E_{1}, \quad \nabla_{\mathrm{E}_{2}} E_{1}=E_{2}, \\
\nabla_{\mathrm{E}_{3}} E_{3}=-E_{1}, \quad \nabla_{\mathrm{E}_{3}} E_{2}=0, \quad \nabla_{\mathrm{E}_{3}} E_{1}=E_{3} .
\end{gathered}
$$


From the above, it can be easily seen that $(\varphi, \xi, \eta, g)$ is a Kenmotsu structure on $M^{3}$. Hence, $\left(M^{3}, \varphi, \xi, \eta, g\right)$ is a 3-dimensional Kenmotsu manifold. By using the above results we can easily obtain,

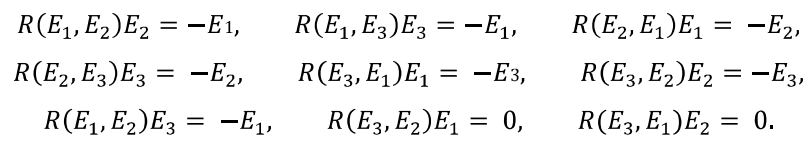

The definition of Ricci tensor in a 3-dimensional manifold implies

$S\left(X_{1}, X_{2}\right)=\sum_{i=1}^{n} g\left(R\left(E_{i}, X_{1}, X_{2}, E_{i}\right)\right.$.

Using the components of the curvature tensor in the above equation we get

$$
\begin{gathered}
S\left(E_{1}, E_{1}\right)=-2, \quad S\left(E_{2}, E_{2}\right)=-2, \quad S\left(E_{3}, E_{3}\right)=-2, \\
S\left(E_{1}, E_{2}\right)=0, \quad S\left(E_{2}, E_{3}\right)=0, \quad S\left(E_{3}, E_{1}\right)=0 .
\end{gathered}
$$

The semi-symmetric metric connection $\widetilde{\nabla}$ is given by (3.1) which gives

$$
\begin{aligned}
& \widetilde{\nabla}_{\mathrm{E}_{1}} E_{2}=0, \quad \widetilde{\nabla}_{\mathrm{E}_{1}} E_{3}=0, \quad \widetilde{\nabla}_{\mathrm{E}_{1}} E_{1}=0, \\
& \widetilde{\nabla}_{\mathrm{E}_{2}} E_{3}=0, \quad \widetilde{\nabla}_{\mathrm{E}_{2}} E_{2}=-2 E_{1}, \quad \widetilde{\nabla}_{\mathrm{E}_{2}} E_{1}=2 E_{2}, \\
& \widetilde{\nabla}_{\mathrm{E}_{3}} E_{3}=-2 E_{1}, \quad \widetilde{\nabla}_{\mathrm{E}_{3}} E_{2}=0, \quad \widetilde{\nabla}_{\mathrm{E}_{3}} E_{1}=2 E_{3} .
\end{aligned}
$$

By using (3.2), we have

$$
\begin{array}{ll}
\tilde{R}\left(E_{1}, E_{2}\right) E_{2}=-2 E_{1}, & \tilde{R}\left(E_{1}, E_{3}\right) E_{3}=-2 E_{1}, \\
\tilde{R}\left(E_{2}, E_{1}\right) E_{1}=-2 E_{2}, & \tilde{R}\left(E_{2}, E_{3}\right) E_{3}=-2 E_{2}, \\
\tilde{R}\left(E_{3}, E_{1}\right) E_{1}=-2 E_{3}, & \tilde{R}\left(E_{3}, E_{2}\right) E_{2}=-2 E_{3}, \\
\tilde{R}\left(E_{1}, E_{2}\right) E_{3}=-2 E_{1}, & \tilde{R}\left(E_{3}, E_{2}\right) E_{1}=0, \\
\tilde{R}\left(E_{3}, E_{1}\right) E_{2}=0 . &
\end{array}
$$

Using the components of the curvature tensor, we have

$$
\begin{array}{lr}
\tilde{S}\left(E_{1}, E_{1}\right)=-4, & \tilde{S}\left(E_{2}, E_{2}\right)=-6, \\
\tilde{S}\left(E_{3}, E_{3}\right)=-6, & \tilde{S}\left(E_{1}, E_{2}\right)=0, \\
\tilde{S}\left(E_{2}, E_{3}\right)=0, & \tilde{S}\left(E_{3}, E_{1}\right)=0 .
\end{array}
$$

Using the above components of the curvature tensor with respect to and (4.18), we get

$$
B_{1}\left(E_{i}\right)+B_{2}\left(E_{i}\right)+B_{4}\left(E_{i}\right)=0, \quad \forall i=1,2,3 \text {. }
$$

Also, using the above components of the Ricci tensor with respect to the semi-symmetric metric connection and (5.8), we get

$$
A_{1}\left(E_{i}\right)+A_{2}\left(E_{i}\right)+A_{3}\left(E_{i}\right)=0, \quad \forall i=1,2,3 .
$$

Thus, this is an example of a 3-dimensional Kenmotsu manifold admitting a semi-symmetric metric connection which is weakly symmetric and weakly Ricci symmetric.

\section{CONCLUSION}

In this study, some geometric properties of weakly symmetric, weakly Ricci symmetric, weakly concircular symmetric, weakly concircular Ricci symmetric and weakly $m$-projectively symmetric Kenmotsu manifolds with respect to a semi-symmetric metric connection have been studied. The conditions for a Kenmotsu manifold to be weakly symmetric and weakly Ricci symmetric with respect to the semi-symmetric metric connection are obtained. Also, we derive the relation between the 1-forms in a weakly concircular symmetric and weakly concircular Ricci symmetric Kenmotsu manifold admitting a semi-symmetric metric connection. A necessary and sufficient condition for the Ricci tensor in a weakly $m$-projectively symmetric Kenmotsu manifold admitting a semi-symmetric metric connection to be Codazzi is obtained and the nature of the scalar curvature in this manifold is discussed.

\section{REFERENCES}

Amur K, Pujar SS (1978) On submanifolds of a Riemannian manifold admitting a metric semi-symmetric connection Tensor, N. S. 32: 35 - 38.

Blair DE (1976) Contact manifolds in Riemannian Geometry Lecture Notes in Math., Springer-Verlag, Berlin, New York.

Binh TQ (1990) On semi-symmetric connections Periodica Math. Hungar. 21(2): $101-107$.

De UC, Biswas SC (1997) On a type of semi-symmetric metric connection on a Riemannian manifold Publ. Inst. Math. 61(75): 90 - 96.

De UC, Ghosh GC (2005) On weakly concircular Ricci symmetric manifolds South East Asian J. Math. and Math. Sci. 3(2): 9 - 15.

Friedman A, Schouten JA (1924) Uber die Geometric der halbsymmetrischen Ubertragung Math. Zs. 21: 211 - 223.

Hayden HA (1932) Subspaces of space with torsion Proc. London Math. Soc. 34: 27 -50.

Kenmotsu K (1972) A class of almost contact Riemannian manifolds Tohuku Math. J. 24: 93 - 103.

Pokhariyal GP, Mishra RS (1971) Curvature tensor and their relativistic significance II,

Yokohama Math. J. 19: 97 - 103. 


\section{Some Results on Weakly Symmetric Kenmotsu Manifolds}

Prakasha DG, Vanli T, Bagewadi CS, Patil DA (2013) Some classes of Kenmotsu manifolds with respect to semi-symmetric metric connection Acta Math. Sinica, English Series 29(7): $1311-1322$.

Prakasha DG, Vikas K (2015) On weak symmetries of Kenmotsu Manifolds with respect to quarter-symmetric metric connection Ann. Math. et Info. 45: 79-90.

Prvanovic M (1975) On pseudosymmetric metric semi-symmetric connections Pub. De L
Institut Math., Nouvelle Serie 18(32): 157 - 164.

Shaikh A, Hui SK (2009) On weakly concircular symmetric manifolds Ann. Sti. Ale. Univ. 30: 219 -224.

Tamassy L, Binh TQ (1992) On weakly symmetric and weakly projective symmetric Riemannian manifolds Coll. Math. Soc. J. Bolyai 56: 663 - 670 .

Yano K (1970) On semi-symmetric metric connection Rev. Roum. Math. Pure Appl. 15: 1579 - 1586. 